\section{Evolution after gene duplication}

\author{
Book review by Tal Pupko, \\ 'Department of Cell Research and \\ Immunology, George S. Wise Faculty \\ of Life Sciences, Tel Aviv University, \\ Israel \\ ${ }^{2}$ National Evolutionary Synthesis Center, \\ Durham, USA
}

(Evolution after Gene Duplication Edited by Katharina Dittmar and David Liberles, Published in hardback by Wiley-Blackwell, 2010 (337 Pages) ISBN-10: 0470593822; ISBN13: 978-0470593820).

Gene duplication is one of the major forces of evolution. Great progress in our understanding of the evolutionary dynamics of gene duplication was achieved in recent years. For example, it is now clear that the paradigm according to which a new copy is generated, travels randomly in sequence space, and by chance find a new function is outdated. Indeed this classical paradigm is not always supported by data and alternative evolutionary models for gene duplication have been suggested.

The book presents our progress in understanding the evolutionary forces, theories, and data in the field of gene duplication. The book is not a comprehensive review of this field. Instead, each chapter sheds light on a specific aspect of gene duplication.

The main strength of the book is that by reading the various chapters together, one can get an excellent overview of the various trajectories of gene duplication research such as system biology, biochemistry, protein structure, and the evolution of alternative splicing. The combination of contributions from expert biochemists, molecular evolutionists, system biologists, and bioinformaticians provides a good outline of the various methodologies that are used for studying gene family evolution. In this respect, the book is very successful in proving a synthesis view of gene duplication research.

The fact that the various chapters were written by different researchers is also the Achilles heel of the book since chapters can contradict each other. As a case in point, chapter 8 (Statistical methods for detecting functional divergence of gene families; $\mathrm{Gu}$ ) starts by stating that: "after a gene duplication, one gene copy maintains the original function, whereas the other copy is free to accumulate amino acid changes toward functional divergence". Such a claim ignores the various alternatives regarding the dynamics of gene duplications that are discussed in lengths in other chapters of the book, e.g., in chapters $1,2,5$, and 6 .

Another nice aspect of the book is that it often allows following the evolution of concepts and scientific thought that shaped gene duplication research. One such example is the review of gene and genome duplications in plants (chapter 15, Gene and genome duplications in plants; Soltis et al.), in which the transformation of ideas is well described: from viewing whole genome duplication (in plants) as evolutionary dead-ends to the current view that questions the existence of true diploids. Changes in our conceptual understanding of gene duplication fate are also provided in chapter 5 (Myths and realities of gene duplication; Hughes and Friedman). Unfortunately, the authors also used this chapter as a platform to include criticism against the vast majority of studies aiming to detect positive Darwinian selection. This criticism is not supported and is largely rejected by the vast majority of molecular evolutionary researchers.

One thing that excited me when reading the book is the current on-going integration of system biology data and techniques to study gene duplication. This is exemplified, for example,
Correspondence: Tal Pupko,

Tel: 972-3-640-7693 - Fax: 972-3-642-2046.

E-mail: talp@post.tau.ac.il

Received for publication: 20 December 2010. Accepted for publication: 20 December 2010.

This work is licensed under a Creative Commons Attribution 3.0 License (by-nc 3.0).

(C) Copyright T. Pupko, 2011

Licensee PAGEPress, Italy

Trends in Evolutionary Biology 2011; 3:e1

doi:10.4081/eb.2011.e1

in the very nice review of Chapter 11 (On the energy and material cost of gene duplication; Wagner), in which research utilizing yeast expression data, proteins half life measurements, population genetic considerations, and gene duplication analyses are integrated to suggest that energetic and material costs impact the fate of duplicated genes. System biology considerations are also described in various other chapters, such as chapters 12 (Fate of Duplication in a network context; Soyer), 13 (Evolutionary and functional aspects of genetic redundancy; Kafri and Pilpel), and 9 (Mapping gene gains and losses among metazoan full genomes using an integrated phylogenetic framework; Tzika, Helaers, and Milinkovitch).

To summarize, Dittmar and Liberles' book is an enjoyable and beneficial read for various scientists: structural biologists, population geneticists, system biologists, molecular evolutionists, bioinformaticians, and systematicians. The collection of various book chapters within a single book provides a whole that is clearly greater than the sum of its parts. 